\title{
Esquizofrenia resistente: Definiciones e Implicancias del concepto de Esquizofrenia Resistente a tratamiento.
}

\section{Treatment resistant schizophrenia: definitions and implications of the Treatment Resistant Schizophrenia concept}

Pizarro-Galleguillos Benjamín ${ }^{1}$, Óscar Waissbluth ${ }^{2}$, Álvaro Cavieres ${ }^{3}$, Pablo R Moya ${ }^{4,5}$ M. Leonor Bustamante ${ }^{6,7}$

\begin{abstract}
Schizophrenia (SZ) is a highly heterogeneous clinical entity. It causes a severe disruption in quality of life, and it imposes a significant burden to society. Antipsychotics are the first line treatment, however up to a $30 \%$ of the patients will present resistance to treatment. Treatment resistant schizophrenia (TRS) could be a neurobiologically distinct disorder and not merely an extremely severe form of SZ. However, there is no consensus in the literature as to the definition of TRS. In the present work we review different definitions of TRS, mainly from clinical guidelines. Furthermore, we discuss therapeutic alternatives for TRS and suggest future perspectives regarding the identification of response predictors and understanding the neurobiology of TRS.
\end{abstract}

Keywords: Treatment resistant, Clozapine, Antipsychotics.

Rev Chil Neuro-Psiquiat 2019; 57 (4): 394-404

Aceptado: 07/01/2020

Recibido: $14 / 08 / 2019$

Los Autores declara no tener ningún conflicto de intereses

1 Interno Medicina, Facultad de Medicina, Universidad de Chile.

2 Clínica Los Tiempos.

3 Departamento de Psiquiatría, Escuela de Medicina, Universidad de Valparaíso.

$4 \quad$ Instituto de Fisiología, Facultad de Ciencias, Universidad de Valparaíso.

5 Centro Interdisciplinario de Neurociencia de Valparaíso CINV, Universidad de Valparaíso.

6 Departamento de Psiquiatría y Salud Mental Norte, Facultad de Medicina, Universidad de Chile.

7 Programa de Genética Humana, Instituto de Ciencias Biomédicas, Facultad de Medicina, Universidad de Chile. 


\section{Introducción}

$\mathrm{L}$ a esquizofrenia (EQZ) es una entidad clínica altamente heterogénea y una mejor caracterización de esta heterogeneidad es necesaria para progresar en la investigación respecto a sus mecanismos causales y posibles tratamientos ${ }^{[1]}$. Una de las variables que presenta importantes diferencias entre los pacientes es la respuesta a tratamiento. Los mecanismos de por qué algunos pacientes presentan síntomas que no mejoran con el tratamiento habitual no están claros, pero varias líneas de evidencia sugieren que podrían representar un subgrupo neurobiológicamente distinto de la enfermedad [1], [2]; Mientras la mayoría de los pacientes con EQZ responden al tratamiento con antipsicóticos (AP) de primera o segunda generación, hasta un tercio de ellos no responden y son caracterizados como resistentes a tratamiento con un rango de valores reportados de $13-43 \%{ }^{[3]-[5]}$. Una importante limitación a la interpretación de estos datos es que no hay consenso en la definición de EQZ resistente al tratamiento (ERT), existiendo diversas definiciones ${ }^{[6]-[8]}$. A continuación, presentaremos una revisión de diferentes propuestas de definición de EQZ resistente, discutiendo las implicancias de ellas para la toma de decisiones clínicas y para la investigación acerca de la neurobiología de la enfermedad.

\section{Esquizofrenia resistente a tratamiento}

Una de las primeras definiciones operacionales de resistencia a tratamiento fue propuesta el año 1988, cuando John Kane et al, demostraron la eficacia de la Clozapina en casos de EQZ que no respondían al tratamiento convencional, la que definieron como la ausencia de respuesta satisfactoria según los puntajes en escalas como BPRS Brief Psychiatric Rating Scale- y CGI -Clinical Global Impression Scale-, luego de tres ensayos terapéuticos, entendidos éstos como tres antipsicóticos a dosis plenas, y tomando como referencia 400-600 mg de clorpromazina ${ }^{[9]}$.

En general, distintas definiciones posteriores se han realizado sobre tres variables principales: indicadores de mejoría por escalas clínicas, número de fármacos ensayados y sus dosis, y el tiempo de tratamiento ${ }^{[6]-[8] \text {, }}$ ${ }^{[10]}$. La tabla 1 presenta un resumen de varias propuestas, detallando los criterios asignados a cada variable.

Al cabo de tres décadas de discusión sobre ERT, persisten significativas controversias entre las diferentes propuestas, revisadas Suzuki ${ }^{[7]}$,primero, que no han demostrado consistencia respecto a la dosis y duración de los ensayos con AP, que varían entre 4 a 6 semanas y con dosis equivalentes a 400-1000 mg de Clorpromazina diarias; por otra parte, que se basan en la medición de los síntomas positivos sin incorporar variables funcionales ni cognitivas de forma operacionalizada.

\section{Tratamiento de la Esquizofrenia resistente con Clozapina.}

A pesar de los diferentes criterios en la definición de ERT, la mayoría de las principales guías clínicas concuerdan en el uso de Clozapina como fármaco de primera línea frente a la resistencia ([12], [13], [15]-[19]. La Clozapina (3-cloro-6-(4-metilpiperazin-1-yl)-11H-benzo ${ }^{[b][1,4]}$ benzodiazepina) ) pertenece a la clase de las dibenzodiazepinas tricíclicas. Clozapina es un agonista inverso de receptores de serotonina tipo 2 (5-HT2A y 5-HT2C) [20]y antagonista de receptores de dopamina tipo 2 (D2, aunque con efecto débil), adrenérgicos 1 y 2 e histaminérgicos H1. La acción de clozapina más allá de aquella sobre receptores de dopamina y serotonina puede explicar otros efectos terapéuticos y secundarios: su antagonismo de receptores muscarínicos M1-5 puede explicar sus efectos anticolinérgicos; la somnolencia observada con clozapina puede explicarse por su antagonismo sobre receptores de his- 


\begin{tabular}{|c|c|c|c|c|c|}
\hline Referencia & $\begin{array}{l}\text { Número, } \\
\text { Tipo y Dosis }\end{array}$ & Tiempo & $\begin{array}{l}\text { Criterios } \\
\text { clínicos de } \\
\text { resistencia }\end{array}$ & Manejo & $\begin{array}{l}\text { Tipo de Estu- } \\
\text { dio }\end{array}$ \\
\hline Kane ${ }^{[9]}$ & $\begin{array}{l}3 \text { fármacos } \\
\text { en } 5 \text { años, }> \\
1000 \text { mg clor- } \\
\text { promazina }\end{array}$ & $\begin{array}{l}6 \text { semanas } \\
\text { cada ensayo }\end{array}$ & $\begin{array}{l}\text { Fun ci o n a - } \\
\text { miento clíni- } \\
\text { co, BPRS >= } \\
\text { 45, CGI míni- } \\
\text { mo de 4; sco- } \\
\text { re de } 4 \text { en dos } \\
\text { dominios del } \\
\text { BPRS: des- } \\
\text { organización } \\
\text { conductual, } \\
\text { delirio perse- } \\
\text { cutorio, com- } \\
\text { portamiento } \\
\text { alucinatorio, } \\
\text { pensamiento } \\
\text { inusual }\end{array}$ & $\begin{array}{l}\text { Clozapina } \\
\text { hasta } 900 \mathrm{mg} / \\
\text { día }\end{array}$ & Estudio $1^{\circ}$ \\
\hline $\begin{array}{l}\text { The Schi- } \\
\text { zophrenia Pa- } \\
\text { tients Outco- } \\
\text { mes Research } \\
\text { Team] }\end{array}$ & $\begin{array}{l}2 \text { fármacos, } \\
\text { uso adecuado }\end{array}$ & & $\begin{array}{l}\text { Persistencia } \\
\text { de síntomas } \\
\text { positivos } \\
\text { clínicamente } \\
\text { significativos }\end{array}$ & $\begin{array}{l}\text { Clozapina } \\
\text { al menos } 8 \\
\text { semana, } 300 \text { - } \\
800 \mathrm{mg} / \text { día }\end{array}$ & $\begin{array}{l}\text { Guía clíni- } \\
\text { ca-Revisión } \\
\text { Sistemática }\end{array}$ \\
\hline \multicolumn{6}{|l|}{$\begin{array}{l}\text { Recom- } \\
\text { mendations } \\
(\text { PORT })^{11}\end{array}$} \\
\hline $\begin{array}{l}\text { American } \\
\text { Psychiatric } \\
\text { Association } \\
(\text { APA })^{[12]}\end{array}$ & $\begin{array}{l}\mathrm{Al} \text { menos } \\
2 \text {, dosis en } \\
\text { rango tera- } \\
\text { péutico }\end{array}$ & 6 semanas & $\begin{array}{l}\text { Poca o nula } \\
\text { respuesta } \\
\text { clínica }\end{array}$ & Clozapina & $\begin{array}{l}\text { Guía } \\
\text { clínica-Revi- } \\
\text { sión Sistemá- } \\
\text { tica }\end{array}$ \\
\hline $\begin{array}{l}\text { European } \\
\text { Medicine } \\
\text { Agency } \\
\text { (EMEA) } \\
{[13]}\end{array}$ & $\begin{array}{l}\text { Al menos 2, } \\
\text { incluyendo } \\
\text { un atípico, en } \\
\text { dosis adecua- } \\
\text { das; una de } \\
\text { las pruebas } \\
\text { debe ser pros- } \\
\text { pectiva }\end{array}$ & $\begin{array}{l}\text { Duración } \\
\text { adecuada }\end{array}$ & $\begin{array}{l}\text { Mejoría } \\
\text { insatisfacto- } \\
\text { ria, habiendo } \\
\text { descartado } \\
\text { consumo de } \\
\text { sustancias }\end{array}$ & Clozapina & $\begin{array}{l}\text { Guía clíni- } \\
\text { ca-Protocolo } \\
\text { Europeo de } \\
\text { Investigación }\end{array}$ \\
\hline
\end{tabular}




\begin{tabular}{|c|c|c|c|c|c|}
\hline Referencia & $\begin{array}{l}\text { Número, } \\
\text { Tipo y Dosis }\end{array}$ & Tiempo & $\begin{array}{l}\text { Criterios } \\
\text { clínicos de } \\
\text { resistencia } \\
\end{array}$ & Manejo & $\begin{array}{l}\text { Tipo de Estu- } \\
\text { dio }\end{array}$ \\
\hline Suzuki ${ }^{[7]},{ }^{[10]}$ & $\begin{array}{l}2 \mathrm{AP} \text {, dosis } \\
\text { equivalente } \\
>600 \mathrm{mg} \text { clor- } \\
\text { promazina }\end{array}$ & 6 semanas & $\begin{array}{l}\text { CGI }>=4, \\
\text { GAF }>50 ; \\
\text { FACT-Sz } \\
<=50\end{array}$ & $\begin{array}{l}\text { No especifi- } \\
\text { cado }\end{array}$ & Revisión \\
\hline $\begin{array}{l}\text { The World } \\
\text { Federation } \\
\text { of Socities } \\
\text { of Biological } \\
\text { Psychiatry } \\
\text { (WFSBP) } \\
{[14],[15]}\end{array}$ & $\begin{array}{l}2 \text { fármacos de } \\
\text { clases quími- } \\
\text { cas diferentes, } \\
\text { al menos } \\
\text { uno atípico } \\
\text { en los } 5 \text { años } \\
\text { previos, a la } \\
\text { dosis reco- } \\
\text { mendada } \\
\text { para cada } \\
\text { fármaco }\end{array}$ & 2-8 semanas & $\begin{array}{l}\text { Persistencia } \\
\text { de sintomato- } \\
\text { logía objeti- } \\
\text { va; habiendo } \\
\text { descartado } \\
\text { otras causas } \\
\text { de falla como } \\
\text { comorbilidad, } \\
\text { uso de sus- } \\
\text { tancias, baja } \\
\text { adherencia a } \\
\text { tratamiento, } \\
\text { polifarmacia }\end{array}$ & $\begin{array}{l}\text { Iniciar Clo- } \\
\text { zapina, dosis } \\
\text { promedio } 400 \\
\text { mg/día }\end{array}$ & $\begin{array}{l}\text { Guía } \\
\text { clínica-Revi- } \\
\text { sión Sistemá- } \\
\text { tica }\end{array}$ \\
\hline $\begin{array}{l}\text { Scottish In- } \\
\text { tercollegiate } \\
\text { Guidelines } \\
\text { Network } \\
\text { (SIGN) }{ }^{[16]}\end{array}$ & $\begin{array}{l}\text { Dos tipos de } \\
\text { fármacos, al } \\
\text { menos uno } \\
\text { de segunda } \\
\text { generación }\end{array}$ & $\begin{array}{l}\text { Duración } \\
\text { adecuada }\end{array}$ & $\begin{array}{l}\text { Falta de } \\
\text { respuesta }\end{array}$ & Clozapina & $\begin{array}{l}\text { Guía Clínica } \\
\text { Nacional-Re- } \\
\text { visión Siste- } \\
\text { mática }\end{array}$ \\
\hline $\begin{array}{l}\text { The National } \\
\text { Institute for } \\
\text { Health and } \\
\text { Care Exce- } \\
\text { llence (NICE) } \\
\text { [17] }\end{array}$ & $\begin{array}{l}\text { Al menos } \\
2 \text { fármacos } \\
\text { incluyendo } \\
\text { un atípico }\end{array}$ & $\begin{array}{l}\text { Duración } \\
\text { adecuada }\end{array}$ & & $\begin{array}{l}\text { Iniciar } \\
\text { Clozapina }\end{array}$ & $\begin{array}{l}\text { Guía clínica } \\
\text { Nacional- Re- } \\
\text { visión Siste- } \\
\text { mática }\end{array}$ \\
\hline $\begin{array}{l}\text { Treatment } \\
\text { response and } \\
\text { resistance } \\
\text { in Psychosis } \\
\text { Group () }{ }^{[8]}\end{array}$ & $\begin{array}{l}>=2 \text { fármacos } \\
>=1 \text { trata- } \\
\text { miento previo } \\
\text { con AP de } \\
\text { larga acción } \\
\text { inyectable }\end{array}$ & $\begin{array}{l}6 \text { semanas v.o } \\
\text { o }>4 \text { meses } \\
\text { parenteral }\end{array}$ & $\begin{array}{l}\text { Reducción } \\
\text { de síntomas } \\
<20 \% \text {; altera- } \\
\text { ción funcio- } \\
\text { nal de acuer- } \\
\text { do a escala } \\
\text { validada; con } \\
\text { adherencia } \\
\text { confirmada } \\
\text { por niveles } \\
\text { séricos del } \\
\text { fármaco }\end{array}$ & $\begin{array}{l}\text { No especifi- } \\
\text { cado }\end{array}$ & $\begin{array}{l}\text { Guía clíni- } \\
\text { ca- Revisión } \\
\text { sistemática }\end{array}$ \\
\hline
\end{tabular}




\begin{tabular}{|c|c|c|c|c|c|}
\hline $\begin{array}{l}\text { Ministerio de } \\
\text { Salud } \\
\text { (MINSAL) }^{[18]}\end{array}$ & $\begin{array}{l}\text { Al menos } 2 \\
\text { fármacos an- } \\
\text { tipsicóticos, } \\
\text { incluyendo } \\
\text { al menos un } \\
\text { AP atípico, en } \\
\text { dosis adecua- } \\
\text { das. }\end{array}$ & 6 semanas & $\begin{array}{l}\text { No hay me- } \\
\text { joría clínica } \\
\text { satisfactoria. } \\
\text { habiendo des- } \\
\text { cartado mala } \\
\text { adherencia, } \\
\text { comorbilidad, } \\
\text { interacción } \\
\text { farmacológi- } \\
\text { ca, consumo } \\
\text { de sustancias. } \\
\text { Se debe opti- } \\
\text { mizar terapia } \\
\text { no farmaco- } \\
\text { lógica. }\end{array}$ & $\begin{array}{l}\text { Iniciar Cloza- } \\
\text { pina y la } \\
\text { Terapia } \\
\text { electro-con- } \\
\text { vulsiva como } \\
\text { alternativas a } \\
\text { los fármacos } \\
\text { antipsicóticos } \\
\text { de uso habi- } \\
\text { tual. }\end{array}$ & $\begin{array}{l}\text { Guía Clínica } \\
\text { Nacional-Re- } \\
\text { visión Siste- } \\
\text { mática }\end{array}$ \\
\hline
\end{tabular}

tamina $\mathrm{H} 1$, y el antagonismo de receptores adrenérgicos al explicaría la hipotensión ortostática observada con clozapina. ${ }^{[21]}$. A diferencia de otros neurolépticos, clozapina tendría menos efectos extra-piramidales [9]. Sin embargo, desde su implementación, se han descrito alteraciones hematológicas importantes tales como agranulocitosis ${ }^{[22],}[23]$. Por este motivo, a partir de 1975 fue retirada en varios países y se mantuvo fuera del mercado y sin financiamiento para la realización de estudios clínicos por más de una década. Más adelante, se permitió su uso solamente en caso de pacientes cuidadosamente seleccionados, incluyendo casos de ERT o síntomas extra piramidales severos ${ }^{[24]}$. Al asociársele un protocolo de vigilancia de efectos hematológicos, disminuyó significativamente la frecuencia de agranulocitosis y la mortalidad por esta causa ${ }^{[9]} \mathrm{La}$ evidencia que sustenta el uso de Clozapina como el fármaco de elección en las ERT se remonta al ya clásico estudio de Kane en $1988^{[5]}$. En este estudio se realizó un ensayo clínico multicéntrico que incluyó 268 pacientes de 16 centros diferentes, en el que se comparó de forma doble-ciego clorpromazina contra Clozapina en ERT que no había respondido a 3 clases distintas de neurolépticos ${ }^{[9]}$. El es- tudio demostró que un 30\% de los pacientes con ERT respondió a Clozapina v/s solo un $4 \%$ que lo hizo con clorpromazina, utilizando el BPRS (Brief Psychiatric Rating Scale), CGI (Clinical Global Impression Scale) y NOBS (Nurse observations calefor inpatient evolution), incluyendo síntomas positivos y negativos ${ }^{[9]}$. Este estudio tenía como antecedentes trabajos previos que mostraban un efecto superior de Clozapina frente otros antipsicóticos, particularmente en pacientes con enfermedades de curso más severo ${ }^{[25]}$, ${ }^{[26]}$, pero la importancia de Kane fue tal que sirvió para que la Clozapina fuese puesta de nuevo en el mercado por la FDA en 1989, con la indicación de usarse en pacientes con ERT $^{[24]}$. Más recientemente, otros protocolos han corroborado estas observaciones entre ellos, CATIE, INTERSEP Y CUTLASS 2, siendo el estudio CATIE el segundo estudio pivotal para avalar el uso de Clozapina, tras el de Kane ${ }^{[24],[27] . ~}$

En 1999, el National Institute of Mental Health de EEUU, al notar que un importante número de estudios sobre nuevos antipsicóticos provenía de ensayos financiados por la industria farmacéutica, contactó al Dr. Jeffrey Lieberman para realizar un gran ensayo multicéntrico para evaluar eficacia 
y tolerabilidad de estos fármacos en condiciones terapéuticas más representativas ${ }^{[24]}$. El estudio CATIE se diseñó en una primera fase para comparar Perfenazina contra nuevos antipsicóticos de manera randomizada y doble ciega. Posteriormente, en una segunda fase aquellos pacientes resistentes fueron randomizados aleatoriamente con Clozapina como alternativa en una rama abierta, versus tratamiento doble ciego con Olanzapina, Quetiapina o Risperidona ${ }^{[28]}$. Entre varios hallazgos, uno de ellos confirmaba a la Clozapina como la droga más efectiva para individuos con pobre respuesta a ensayos previos con otros antipsicóticos, a pesar de estar asociada a efectos adversos serios, tales como el aumento de peso y complicaciones metabólicas, y aun cuando la disminución en los síntomas medidos por medio de PANSS fueron modestos ${ }^{[28]}$. Por otra parte, con respecto a otros dominios funcionales, los datos de este estudio muestran que ninguno de los tratamientos produce una mejoría significativa en los síntomas cognitivos ni en la calidad de vida ${ }^{[29],[30]}$.

A pesar de la robusta evidencia de grandes estudios observacionales y de estudios de rama abierta que han apoyado el uso de Clozapina como Gold Standard en ERT, actualmente existe cierta controversia en torno a su efectividad debido a que el 2016 se publicaron dos trabajos con evidencia contradictoria $^{[31]}$. Por un lado, un Network Metanalysis publicado por Samara et al, 2016 ${ }^{[32]}$ solo demostró beneficios pequeños de Clozapina, Olanzapina y Risperidona en síntomas generales en ERT, sin un beneficio claro de Clozapina sobre otros AP. Por otra parte, un segundo Meta-análisis publicado por Siskind et al, 2016 ${ }^{[33]}$, demostró superioridad de Clozapina versus el resto de AP de $1^{\circ}$ y $2^{\circ}$ Generación, para reducción de síntomas positivos en el corto y largo plazo en ERT, además de demostrar que mayor severidad inicial se correlacionaba con mejor respuesta a Clozapina. Sin perjuicio de lo anterior, como
Taylor $2017^{[31]}$, señala, aún con estos resultados contradictorios, 30 años de evidencia acumulada, aún sustentan el uso de Clozapina como Gold standard en ERT. Interesantemente, Taylor recalca, que las diferencias obtenidas en estos meta- análisis podrían corresponder a diferencias metodológicas, particularmente debido a la heterogeneidad en las definiciones de ERT utilizadas, destacando así la importancia de adoptar criterios similares para su definición.

\section{Resistencia y Pronóstico}

La EQZ es una enfermedad crónica, en donde hasta el $80-90 \%$ de los pacientes presentaran algún tipo de disfunción social o laboral persistente ${ }^{[34]}$. En ese sentido, la EQZ presenta un curso episódico con recaídas que pueden tener una severidad variable. Sin perjuicio de lo anterior, La EQZ es una enfermedad crónica, en donde hasta el $80-90 \%$ de los pacientes presentaran algún tipo de disfunción social o laboral persistente $^{[34]}$. Considerando esto, algunos autores han usado la cronicidad como un marcador de resistencia, definido como el número de hospitalizaciones o la existencia de hospitalizaciones prolongadas ${ }^{[4]}$. En relación a las recaídas, un estudio reciente de Kesserwani kadra et. $\mathrm{Al}^{[35]}$ que incluyó 3651 individuos encontró asociación del uso de Clozapina y una disminución de re hospitalizaciones comparándolos con quienes usaban otros AP en monoterapia. Esto se sostuvo aun encontrándose los pacientes que fueron tratados con Clozapina más gravemente enfermos, con un estado funcional más deficiente y una psicopatología más grave al inicio de la prescripción. Considerar la tasa de recaídas tiene un impacto económico, como observaron Kennedy et. $\mathrm{a}^{[36]}$. quienes mostraron que del costo de la EQZ en EEUU fue de 34 mil millones de dólares anuales en costos médicos, considerando un costo anual por paciente entre 15500 y 22300 dólares por paciente, el cual se triplica en el caso de la ERT ${ }^{[37]}$. 


\section{Discusión}

Las investigaciones acerca de la eficacia de los antipsicóticos en EQZ han definido la resistencia a tratamiento de forma heterogénea, lo que limita la validez de las guías basadas en la evidencia. Sin embargo, es posible encontrar algunos elementos comunes a todas las definiciones.

En primer lugar, casi todos los estudios comparan la respuesta sólo en términos de síntomas positivos. El rol central de los síntomas positivos dice relación con el efecto de estos fármacos, que es claramente superior en este dominio sintomático respecto a otros, así como en la relevancia de ellos para la discapacidad de los pacientes [2]. Aunque existe creciente evidencia de que los síntomas cognitivos y negativos pueden tener un rol pronóstico a largo plazo, hasta ahora no hay evidencia de que los AP puedan tener un rol decisivo en aliviarlos. Esto parece ser la causa de por qué incluso estudios contemporáneos, realizados en un período de mayor conciencia respecto al impacto de estos otros dominios sintomáticos, siguen enfocándose casi exclusivamente en los síntomas positivos. No obstante, dado el impacto de los síntomas cognitivos en la funcionalidad de los pacientes, es necesario que clínicos e investigadores, consideren la importancia de los mismos para la búsqueda de futuras intervenciones.

Un segundo aspecto que emerge con claridad, es que las guías recomiendan decididamente la Clozapina en el manejo de las ERT. Aunque los plazos para definir su inicio son variables, estos fluctúan alrededor de las 6 semanas. Contrastando con esta recomendación, reportes de la literatura muestran que en la práctica existe un retraso importante en la instalación de este AP, que promedia los cuatro años [38]. Esta cifra supera a cualquiera de las recomendaciones y se asocia al uso, en promedio, de 5 AP previo a la instalación de Clozapina, alcanzando además dosis mayores de las recomendadas en las guías clínicas [38]. Este gran retraso también fue encontrado en otros estudios como el de Wheeler en nueva Zelanda donde el tiempo previo al inicio de la administración promedia los 10 años [39]. Dentro de la demora en la instalación de Clozapina, se han identificado ciertas barreras que involucran tanto al médico prescriptor como a las instituciones. Farooq et al en una revisión sistemática desde 1972 a marzo del 2018 seleccionó 15 artículos relacionados con barreras para el uso de Clozapina en pacientes con ERT y encontró que las principales causas se relacionaban con la extracción de sangre obligatorio ,miedo a los efectos secundarios graves, falta de familiaridad de uso del fármaco, falta de claridad en los diagnósticos, dificultad para identificar/seleccionar a los pacientes adecuados, fragmentación en el servicio (toma de examen, entrega del resultado receta y entrega del fármaco) entrenamiento deficiente del personal de salud [40]. La resistencia de los tratantes a indicar Clozapina no está fundamentada en la realidad, como concluye un estudio que analizó la mortalidad en más de 14.000 personas con trastornos mentales severos y que identificó una fuerte asociación entre haber recibido Clozapina y una reducción en la mortalidad [41]. Los autores indican que el tamaño de este efecto protector no se explica solo por el hecho de recibir un seguimiento hematológico, y que se explicaría tanto por una reducción en el riesgo de suicidio como por una reducción en la mortalidad por causas médicas.

La severa disfunción que se asocia a la psicosis, junto con el bien establecido beneficio de Clozapina, hacen patente la necesidad de identificar predictores de respuesta que puedan ser medidos tempranamente en el curso del tratamiento con antipsicóticos. Dado que la identificación de los pacientes resistentes requiere un período de observación de varias semanas, con dosis plenas de 
AP, previo a definir un tratamiento adecuado a las características específicas de estos pacientes, ellos conviven con síntomas psicóticos que pueden traer un deterioro severo de su calidad de vida, incluyendo el riesgo de suicidio; junto con ello, se ven expuestos a potenciales efectos colaterales de fármacos en dosis altas. Se conocen factores ambientales que pueden contribuir a la ERT, como por ejemplo el tiempo de duración de la psicosis sin tratamiento o el abuso de sustancias. También se ha identificado un rol importante de factores genéticos [42], [43]. Sin embargo, las bases biológicas no se conocen con precisión y no hay biomarcadores ni predictores clínicos claros para identificar precozmente a los pacientes que requerirán Clozapina. Interesantemente, una revisión de 2018 que reúne 92 estudios farmacogenéticos sobre variaciones en la eficacia de AP muestra que incluye solo 19 individuos de ancestría hispánica de un total de 9600 pacientes [44]. Dado que se ha demostrado una asociación entre la ancestría y parámetros farmacocinéticos y farmacodinámicos, esta carencia pone énfasis en la necesidad de desarrollar estudios en nuestra población chilena que contribuyan a la toma de decisiones de salud relevantes para los individuos y la población.

Más aún, se han propuesto nuevas perspectivas en torno a la clasificación de la ERT. Recientemente, Kinon et al [45], plantea la posibilidad de dividir a los pacientes con ERT en primarios o secundarios, es decir en aquellos cuya resistencia se manifiesta antes de 5 los años de tratamiento después de los 5 años. Además, Kinon, plantea la categoría de Ultra resistencia a tratamiento, definida como aquellos pacientes con ERT que no responden a Clozapina. Bajo la misma lógica, el grupo de Lee et al., proponen la distinción entre "respondedor a AP", "respondedor a Clozapina", y "resistente a Clozapina" [2]. Ambas propuestas abren, nuevamente, la discusión de si este grupo corresponde a un espectro de severidad, o más bien, a un grupo categorialmente distinto de EQZ con una neurobiología y arquitectura genética distinta. De este modo, los objetivos de estas nuevas propuestas están en línea con la intención del Research Domain Criteria (RDoC) de modificar la nosología de las enfermedades psiquiátricas usando variables neurobiológicas [46]

En suma, aunque no existen criterios unificados en torno a la definición de la ERT, este problema clínico puede ser abordado por diversas estrategias. Es por esto que una aproximación crítica a esta definición y sus implicancias, permitirá que los clínicos optimicen el tratamiento de este grupo de pacientes, mejorando su pronóstico y reduciendo costos; al mismo tiempo permitirá a los investigadores, profundizar y ampliar el espectro de las investigaciones en torno a las causas y los tratamientos de la esquizofrenia.

\section{Resumen}

La esquizofrenia (EQZ) es una entidad clínica altamente heterogénea. Determina un severo impacto en la calidad de vida de los pacientes y un alto costo para la sociedad. Los antipsicóticos son la primera línea de tratamiento, sin embargo, hasta un tercio de los pacientes presentaran una esquizofrenia resistente a tratamiento (ERT). Se ha propuesto que la ERT podría corresponder a un grupo neurobiológicamente distinto de la enfermedad con una arquitectura genética particular y no solo al extremo del 
espectro de severidad de la misma. A pesar de ello, actualmente no existe consenso en la literatura en torno a la definición de ERT. En este trabajo presentamos una revisión de diferentes definiciones de ERT centrándonos principalmente en las guías clínicas publicadas. Además se discuten las alternativas terapéuticas en ERT y, finalmente, se proponen perspectivas futuras en torno a la necesidad de desarrollar predictores de respuesta a antipsicóticos de primera y segunda línea, así como también la posibilidad de comprender la neurobiología de la ERT.

Palabras claves: Resistencia a tratamiento, Clozapina, Antipsicóticos

\section{Referencias Bibliográficas}

1. [A. L. Gillespie, R. Samanaite, J. Mill, A. Egerton, and J. H. MacCabe, "Is treatment-resistant schizophrenia categorically distinct from treatment-responsive schizophrenia? A systematic review," BMC Psychiatry, 2017.

2. J. Lee et al., "Subtyping schizophrenia by treatment response: Antipsychotic development and the central role of positive symptoms," Canadian Journal of Psychiatry. 2015.

3. L. B. Dixon, A. F. Lehman, and J. Levine, "Conventional antipsychotic medications for schizophrenia.," Schizophr. Bull., vol. 21, no. 4, pp. 56777, 1995.

4. H. Elkis, "Treatment-Resistant Schizophrenia," Psychiatr. Clin. North Am., vol. 30, no. 3, pp. 511-533, 2007.

5. M. Herrera-Estrella and K. Luna, "Clozapina: una revisión," Psiquiatr. Biol., vol. 23, no. 3, pp. 87-92, 2016.

6. A. Barquero-Madrigal, "Esquizofrenia resistente al tratamiento: ¿hemos tomado una decisión? Treatment-resistant schizophrenia: have we made a decision?," vol. 55, no. 2, pp. 85-92, 2017.

7. T. Suzuki et al., "Defining treatment-resistant schizophrenia and response to antipsychotics: A review and recommendation," Psychiatry Res., vol. 197, no. 1-2, pp. 1-6, 2012.

8. O. Agid, A. Board, C. J. Johnson, E.
Lilly, U. S. Company, and E. L. Canada, "Treatment resistant schizophrenia: Treatment Response and Resistance in Psychosis ( TRRIP ) working group consensus guidelines on diagnosis and terminology," vol. 174, no. 666, pp. 216-229, 2018.

9. J. Kane, "Clozapine for the Treatment-Resistant Schizophrenic," Arch. Gen. Psychiatry, vol. 45, no. 9, p. 789, 1988.

10. T. Suzuki et al., "Treatment resistant schizophrenia and response to antipsychotics: A review," Schizophr. Res., vol. 133, no. 1-3, pp. 54-62, 2011.

11. J. Kreyenbuhl, R. W. Buchanan, F. B. Dickerson, and L. B. Dixon, "The schizophrenia patient outcomes research team (PORT): Updated treatment recommendations 2009," Schizophrenia Bulletin. 2010.

12. L. B. Dixon and D. O. Perkins, "Treatment of Patients With Schizophrenia Second Edition," Am. Psychiatr. Assoc., no. February, pp. 1-184, 2010.

13. European Medicines Agency, "Guideline on clinical investigation of medicinal products, including depot preparations in the treatment of schizophrenia," vol. 44, no. September, pp. 1-24, 2012.

14. A. Hasan et al., "World Federation of Societies of Biological Psychiatry (WFSBP) Guidelines for Biological Treatment of Schizophrenia, Part 2: Update 2012 on the long-term treat- 
ment of schizophrenia and management of antipsychotic-induced side effects," World J. Biol. Psychiatry, vol. 14, no. 1, pp. 2-44, 2013.

15. A. Hasan et al., "World Federation of Societies of Biological Psychiatry (WFSBP) Guidelines for Biological Treatment of Schizophrenia, Part 1: Update 2012 on the acute treatment of schizophrenia and the management of treatment resistance," World J. Biol. Psychiatry, vol. 13, no. 5, pp. 318378, 2012.

16. Healthcare Improvement Scotland, "Management of schizophrenia: A key to Evidence Statements and grades of recommendations.," Sign, no. March, pp. 1-71, 2013.

17. National Institute of Health and Clinical Excellence, "Psychosis and schizophrenia in adults," NICE Guidel. treament Manag., no. February, pp. 74-80, 2014.

18. Ministerio de Salud Subsecretaria de Salud, "Guía Clínica para el tratamiento de personas desde primer episodio de Esquizofrenia," Guías Clínicas MINSAL, pp. 1-99, 2009.

19. D. Addington, S. Abidi, I. Garcia-Ortega, W. G. Honer, and Z. Ismail, "Canadian Guidelines for the Assessment and Diagnosis of Patients with Schizophrenia Spectrum and Other Psychotic Disorders," Can. J. Psychiatry, vol. 62, no. 9, pp. 594-603, 2017.

20. L. C. Sullivan, W. P. Clarke, and K. A. Berg, "Atypical antipsychotics and inverse agonism at 5-HT2 receptors.," Curr. Pharm. Des., vol. 21, no. 26, pp. 3732-8, 2015.

21. "National Center for Biotechnology Information. PubChem Database. Clozapine, $\mathrm{CID}=135398737$, , p. $\mathrm{CID}=135398737$.

22. R. W. Griffith and K. Saameli, "Clozapine and Agranulocytosis," Lancet, vol. 306, no. 7936, p. 657, Oct. 1975.

23. A. de la Chapelle, C. Kari, M. Nurminen, and S. Hernberg, "Clozapine-induced agranulocytosis - A genetic and epidemiologic study," Hum. Genet., 1977.

24. O. Freudenreich, H. E. Brown, and D. J. Holt, 28 - Psicosis y esquizofrenia, Second Edi. Elsevier Espa8\#241;a, S.L.U., 2018.

25. J. Claghorn et al., "The risks and benefits of clozapine versus chlorpromazine.," J. Clin. Psychopharmacol., vol. 7, no. 6, pp. 377-84, Dec. 1987.

26. K. A. Fischer-Cornelssen and U. J. Ferner, "An example of European multicenter trials: multispectral analysis of clozapine.," Psychopharmacol. Bull., vol. 12, no. 2, pp. 34-9, Apr. 1976.

27. J. A. Inchauspe Aróstegui and M. Á. Valverde Eizaguirre, "Creer en la clozapina: fe y evidencias," Rev. la Asoc. Española Neuropsiquiatría, vol. 38, no. 133, pp. 239-262, 2018.

28. M. S. Swartz et al., "Special Section on Implications of CATIE: What CATIE Found: Results From the Schizophrenia Trial," Psychiatr. Serv., vol. 59 , no. 5, pp. 500-506, 2008.

29. M. D. Marvin S. Swartz et al., "Effects of antipsychotic medications on psychosocial functioning in patients with chronic schizophrenia: findings from the NIMH CATIE study," Am. J. Psychiatry, 2007.

30. R. S. E. Keefe et al., "Neurocognitive effects of antipsychotic medications in patients with chronic schizophrenia in the CATIE trial," Arch. Gen. Psychiatry, 2007.

31. D. M. Taylor, "Clozapine for Treatment-Resistant Schizophrenia: Still the Gold Standard?," CNS Drugs, vol. 31, no. 3, pp. 177-180, 2017.

32. M. T. Samara et al., "Efficacy, accep- 
tability, and tolerability of antipsychotics in treatment-resistant schizophrenia: A network meta-analysis," JAMA Psychiatry, vol. 73, no. 3, pp. 199-210, 2016.

33. D. Siskind, L. McCartney, R. Goldschlager, and S. Kisely, "Clozapine v. first- and second-generation antipsychotics in treatment-refractory schizophrenia: Systematic review and meta-analysis," Br. J. Psychiatry, vol. 209, no. 5, pp. 385-392, 2016.

34. H. Y. Meltzer, "Commentary: Defining Treatment Refractoriness in Schizophrenia," Schizophr. Bull., vol. 16, no. 4, pp. 563-565, Jan. 1990.

35. J. Kesserwani et al., "Risk of readmission in patients with schizophrenia and schizoaffective disorder newly prescribed clozapine.," J. Psychopharmacol., vol. 33, no. 4, pp. 449-458, Apr. 2019.

36. J. L. Kennedy, C. A. Altar, D. L. Taylor, I. Degtiar, and J. C. Hornberger, "The social and economic burden of treatment-resistant schizophrenia: a systematic literature review.," Int. Clin. Psychopharmacol., vol. 29, no. 2, pp. 63-76, Mar. 2014.

37. J. L. Gören, A. J. Rose, E. G. Smith, and J. P. Ney, "The Business Case for Expanded Clozapine Utilization," Psychiatr. Serv., vol. 67, no. 11, pp. 1197-1205, Nov. 2016.

38. O. D. Howes, F. Vergunst, S. Gee, P. McGuire, S. Kapur, and D. Taylor, "Adherence to treatment guidelines in clinical practice: study of antipsychotic treatment prior to clozapine initiation.," Br. J. Psychiatry, vol. 201, no. 6, pp. 481-5, Dec. 2012.

39. A. J. Wheeler, "Treatment pathway and patterns of clozapine prescribing for schizophrenia in New Zealand.," Ann. Pharmacother., vol. 42, no. 6, pp. 852-60, Jun. 2008.
40. S. Farooq, A. Choudry, D. Cohen, F. Naeem, and M. Ayub, "Barriers to using clozapine in treatment-resistant schizophrenia: systematic review," BJPsych Bull., 2019.

41. R. D. Hayes et al., "The effect of clozapine on premature mortality: an assessment of clinical monitoring and other potential confounders.," Schizophr. Bull., vol. 41, no. 3, pp. 64455, May 2015.

42. M. J. Arranz and J. de Leon, "Pharmacogenetics and pharmacogenomics of schizophrenia: a review of last decade of research.," Mol. Psychiatry, vol. 12, no. 8, pp. 707-47, Aug. 2007.

43. D. J. Müller et al., "Familial occurrence of tardive dyskinesia," Acta Psychiatr. Scand., vol. 104, no. 5, pp. 375-379, Jul. 2008.

44. K. Yoshida and D. J. Müller, "Pharmacogenetics of Antipsychotic Drug Treatment: Update and Clinical Implications," 2018.

45. B. J. Kinon, "The group of treatment resistant schizophrenias. Heterogeneity in treatment resistant schizophrenia (TRS)," Front. Psychiatry, vol. 10, no. JAN, pp. 1-6, 2019.

46. D. C. Javitt, "Biotypes in Psychosis: Has the RDoC Era Arrived?," Am. J. Psychiatry, vol. 173, no. 4, pp. 313-4, Apr. 2016.

Correspondencia:

María Leonor Bustamante, Avenida la Paz 1103, Recoleta, mbustamante@med.uchile.cl, 22978601 